\title{
Article
}

\section{Separation and Recovery of Eu(III) and Y(III) with Solvent Impregnated Resin Coated by Crosslinked Chitosan}

\author{
Syouhei NISHIHAMA*, Akane KAWASAKI and Kazuharu YOSHIZUKA \\ Department of Chemical Engineering, The University of Kitakyushu, Hibikino 1-1, Kitakyushu 808-0135, Japan
}

(Manuscript received May 28, 2014; Accepted July 3, 2014)

\begin{abstract}
Separation of europium $\left(\mathrm{Eu}^{3+}\right)$ and yttrium $\left(\mathrm{Y}^{3+}\right)$, using a solvent impregnated resin (SIR) containing 2ethylhexyl phosphonic acid mono-2-ethylhexyl ester, coated by water-soluble chitosan crosslinked by glutaraldehyde, was investigated. Coating of the SIR successfully suppressed leakage of the extractant during adsorption processing. Adsorption of $\mathrm{Eu}^{3+}$ with both coated and uncoated SIRs was progressed with Langmuir mechanism, and the maximum adsorption amount of $\mathrm{Eu}^{3+}$ at equilibrium $\mathrm{pH}=2.27$ was $0.294 \mathrm{~mol} / \mathrm{kg}$ with coated SIR and $0.310 \mathrm{~mol} / \mathrm{kg}$ with uncoated SIR. Decrease in the maximum adsorption amount by coating SIR with polymer can be suppressed rather than the other coated SIRs previously reported. The coated SIR was then applied to column separation of $\mathrm{Eu}^{3+}$ and $\mathrm{Y}^{3+}$. Complete separation of the metals could be achieved by gradient elution with a gradual increase in the concentration of a dilute nitric acid. The coated SIR could also be reused for repeated adsorption-elution processing.
\end{abstract}

Keywords: SIR, Coating, Separation, Gradient Elution

\section{Introduction}

Rare earth metals are widely used as raw materials for fluorescent lamps, vehicle catalysts, cathode ray tubes, and permanent magnet motors. In Japan, most rare earth metals are imported from abroad and thus the development of effective recovery processes of rare earth metals from the waste is an attractive issue from the view point of resource sustainability. Spent fluorescent lamps are one of the attractive materials, since the lamps contain phosphor powders based on the rare earth metals. A few hydrometallurgical separation and recovery of the rare earth metals from spent fluorescent lamps were however reported ${ }^{1,2)}$.

Solvent extraction is presently one of the major industrialscale techniques for the separation and recovery of metals and has the advantage for easily applicable to large scale continuous operation. However, one disadvantage is that the organic diluent, used to dissolve both the extractant and the extracted species, may be lost into the aqueous phase owing to its aqueous solubility. Combination of solvent extraction with adsorption and/or ion exchange has been therefore developed as a second-generation extraction system. Solvent impregnated resins (SIRs) have been widely investigated among the techniques, due to their advantage of simple preparation ${ }^{3-8)}$.

Disadvantage of the SIRs is the leakage of the extractant from the resin during adsorption process, because the extractant is not chemically immobilized within the resin. Coating of SIR with thin polymeric film to prevent the leakage of the extractant has therefore recently been reported ${ }^{9-13)}$. A review paper concluded that a crosslinked water-soluble polymer, such as polyvinyl alcohol (PVA), is a suitable polymeric film, because the extractant impregnated is to be dissolved into organic solvent when the coating is carried out in organic solution $^{14)}$. In our previous work, an SIR impregnated with 2ethylhexyl phosphonic acid mono-2-ethylhexyl ester (PC-88A) within a methacrylic ester copolymer resin coated with crosslinked polyvinyl alcohol (PVA) was developed for applying for the separation of lanthanum and cerium contained in spent vehicle catalysts ${ }^{15}$. Coating of the SIR with

\footnotetext{
* Corresponding author

E-mail: nishihama@kitakyu-u.ac.jp
} 
crosslinked PVA successfully suppressed the leakage of the extractant during adsorption. The coated SIR could also separate the two rare earth metals by chromatographic operation using gradient elution with a gradual increase in the concentration of dilute nitric acid.

In the present work, separation of $\mathrm{Eu}^{3+}$ and $\mathrm{Y}^{3+}$, existing in red phosphor materials, using a coated SIR impregnating PC$88 \mathrm{~A}$ in a methacrylic ester copolymer resin is investigated. Crosslinked water-soluble chitosan was used for coating SIR, to investigate the feasibility of chitosan as a coating material. The effect of the concentration of chitosan on both the adsorption of the metals and the leakage of the extractant were investigated. The coated SIR was then applied to the chromatographic separation of $\mathrm{Eu}^{3+}$ and $\mathrm{Y}^{3+}$, using gradient elution mode, to achieve complete separation of the metals.

\section{Experimental}

\subsection{Reagents}

Commercial extractant, 2-ethylhexylphosphonic acid mono2-ethylhexyl ester (marketed as PC-88A by Daihachi Chemical Industry Co., Ltd., Osaka, Japan), was used as received. Diaion HP2MG, a methacrylic ester copolymer containing no functional group, was kindly supplied by Nippon Rensui Co., Ltd. (Tokyo, Japan). Water-soluble chitosan (Yang Chito 100) was supplied by Yeongdeok Chitosan (Yeongdeok, Korea). Average molecular weight of the water-soluble chitosan, determined by gel permeation chromatography (Shodex GPC101) was 19490 (average polymerization degree was 121). All other organic and inorganic reagents were supplied by Wako Pure Chemical Industries, Ltd. (Osaka, Japan), as analyticalgrade reagents.

\subsection{Preparation and Coating of SIR}

The SIR was prepared by the following method ${ }^{15)}$. HP2MG was washed with methanol and dried in vacuo. Washed HP2MG $\left(2.5 \times 10^{-3} \mathrm{~kg}\right)$ was then immersed for overnight in a $0.1 \mathrm{~mol} / \mathrm{dm}^{3} \mathrm{PC}-88 \mathrm{~A}$ - toluene solution $\left(50 \mathrm{dm}^{3}\right)$. The toluene was then removed by evaporation and the SIR was dried in vacuo for $24 \mathrm{~h}$. The amount of PC-88A impregnated in the SIR was $1.24 \mathrm{~mol} / \mathrm{kg}$. The uncoated SIR is simply abbreviated as SIR hereafter.

Coating of SIR was conducted using chitosan crosslinked by glutaraldehyde. The prepared SIR $\left(3 \times 10^{-3} \mathrm{~kg}\right)$ was immersed in an aqueous solution $\left(50 \mathrm{dm}^{3}\right)$ containing 1 or $3 \mathrm{wt} \%$ chitosan and the mixture was shaken for $24 \mathrm{~h}$. The resultant SIR was filtered and re-suspended in $0.32 \mathrm{~mol} / \mathrm{dm}^{3} \mathrm{H}_{2} \mathrm{SO}_{4}$ aqueous solution $\left(10 \mathrm{dm}^{3}\right)$ for 1 h. $0.32 \mathrm{~mol} / \mathrm{dm}^{3}$ glutaraldehyde aqueous solution $\left(10 \mathrm{dm}^{3}\right)$ was then added, followed by shaking the mixture for $24 \mathrm{~h}$, to complete crosslinking. The coated SIR obtained was filtered, washed with deionized water, and then dried.

Surfaces of the SIRs (HP2MG, SIR, and coated SIR) were observed with a scanning electron microscope (SEM, Keyence VE-8800) without spatter coating.

\subsection{Batchwise Adsorption}

Aqueous solutions of $\mathrm{Eu}^{3+}$ and $\mathrm{Y}^{3+}$ were prepared by dissolving each nitrate salt in deionized water. The concentration of $\mathrm{Eu}^{3+}$, in the case of single metal systems for $\mathrm{pH}$ dependence experiments, was set at $1.0 \mathrm{mmol} / \mathrm{dm}^{3}$, while the concentration of $\mathrm{Eu}^{3+}$ was varied from 0.001 to 40 $\mathrm{mmol} / \mathrm{dm}^{3}$ for adsorption isotherm experiments. In the binary metal system containing $\mathrm{Eu}^{3+}$ and $\mathrm{Y}^{3+}$, the concentrations of both metals were set at $1.0 \mathrm{mmol} / \mathrm{dm}^{3}$. The $\mathrm{pH}$ was adjusted by adding the appropriate amount of nitric acid solution or sodium hydroxide solution to the aqueous solution. SIR or coated SIR $\left(5.0 \times 10^{-5} \mathrm{~kg}\right)$ was added to $10 \mathrm{dm}^{3}$ of aqueous solution, and the mixture was shaken at $298 \mathrm{~K}$ for $48 \mathrm{~h}$. After filtration, the equilibrium $\mathrm{pH}$ was measured. The concentrations of metal ions were determined by an inductively coupled plasma atomic emission spectrometer (ICP-AES, Shimadzu ICPS-7000). The adsorption amount of metal ion, $q$, is defined by equation (1):

$$
q=\frac{\left([\mathrm{Ln}]_{\mathrm{feed}}-[\mathrm{Ln}]\right) \cdot L}{w}
$$

where $[\mathrm{Ln}]_{\text {feed }}$ and $[\mathrm{Ln}]$ represent the initial and equilibrium concentrations of the rare earth metal in the aqueous phase, and $L$ and $w$ are the volume of aqueous solution and weight of SIR or coated SIR, respectively. Leakage of the extractant was determined by measuring phosphorous concentration in the aqueous solution after the adsorption by ICP-AES.

\subsection{Chromatographic Separation of $\mathrm{Eu}^{3+}$ and $\mathrm{Y}^{3+}$}

Coated SIR with 3 wt $\%$ chitosan was applied to chromatographic separation of $\mathrm{Eu}^{3+}$ and $\mathrm{Y}^{3+}$. Two kinds of operation modes, frontal analysis and gradient elution modes, were applied, using the column setup shown in Fig. 1. In the case of the frontal analysis mode, $2.0 \times 10^{-3} \mathrm{~kg}$ of the coated SIR (wet volume $2.0 \mathrm{dm}^{3}$ ) was packed into a column, $8 \mathrm{~mm}$ diameter and $100 \mathrm{~mm}$ height, together with quartz wool to be sandwiched and washed with deionized water. The spent fluorescent lamp generally includes red, blue, and green phosphor materials. In the present work, the red phosphor materials is focused, and an aqueous feed solution was prepared at $\mathrm{pH}=5.3$ and $0.5 \mathrm{mmol} / \mathrm{dm}^{3}$ of $\mathrm{Eu}^{3+}$ and 13.3 $\mathrm{mmol} / \mathrm{dm}^{3}$ of $\mathrm{Y}^{3+}$. The aqueous feed solution was fed upwards to the column at a flow rate of $0.1 \mathrm{dm}^{3} / \mathrm{min}$ (space velocity, S.V. $=3.0 \mathrm{~h}^{-1}$ ), using a dual plunger pump (Flow KP-11) until the adsorption reached saturation. After saturating the adsorption of metals, deionized water was fed to the column to wash out residual feed solution for more than $24 \mathrm{~h}$. The loaded metal ions were then eluted by $1.0 \mathrm{~mol} / \mathrm{dm}^{3}$ nitric acid solution.

In the case of gradient elution mode, the adsorption was carried out by the same conditions with the frontal analysis mode. After the adsorption was saturated, the column was washed with deionized water. Another column, $8 \mathrm{~mm}$ diameter 
and $300 \mathrm{~mm}$ height, packed with $6.0 \times 10^{-3} \mathrm{~kg}$ of fresh coated SIR (wet volume $6.0 \mathrm{dm}^{3}$ ) was then connected over the adsorption column as a development column. The loaded metals were then eluted by nitric acid solution, with a concentration gradually changed from 0.2 to $1.0 \mathrm{~mol} / \mathrm{dm}^{3}$ by mixing $1.0 \mathrm{~mol} / \mathrm{dm}^{3}$ nitric acid solution and deionized water with a low pressure gradient former (EYELA LPG-1000). The effluents were collected with a fraction collector (EYELA DC1500). The $\mathrm{pH}$ and metal ion concentrations were measured by $\mathrm{pH}$ meter and ICP-AES, respectively. The bed volume of the effluent is defined as:

$$
\text { Bed Volume }=\frac{v \cdot t}{V}
$$

where $v, t$ and $V$ are the volumetric flow rate of solution, the time for which the feed solution was applied, and the wet volume of the adsorbent, respectively.

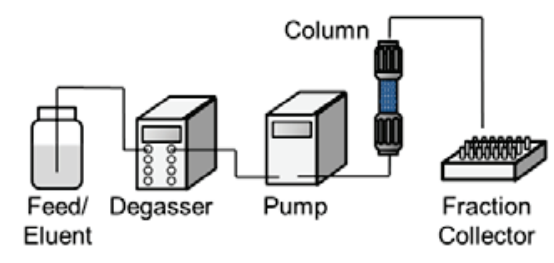

(a) Column setup for frontal analysis mode

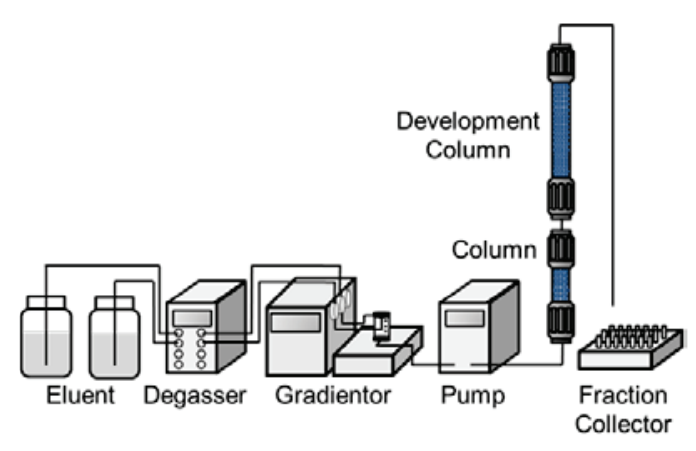

(b) Column setup for gradient elution mode

Fig. 1 Schematic flowsheet for the column adsorption apparatus setup.

\section{Results and Discussion \\ 3.1 Characterization of SIRs}

Figure 2 shows the SEM images of HP2MG, SIR, and SIR coated by $3 \mathrm{wt} \%$ chitosan. No significant change in the surface morphology is seen on HP2MG, SIR, and coated SIR and smooth surface is observed (a-1, b-1, and c-1). When the surface is observed at 5000-power, micropores of the resin are seen on the surface of the HP2MG (a-2) and of the SIR (b-2). The micropores are however disappeared, when the SIR was coated by crosslinked chitosan, although small deficit of the polymer layer was sometimes seen on the surface (c-2). The SIR is therefore successfully coated by water-soluble chitosan crosslinked by glutaraldehyde.
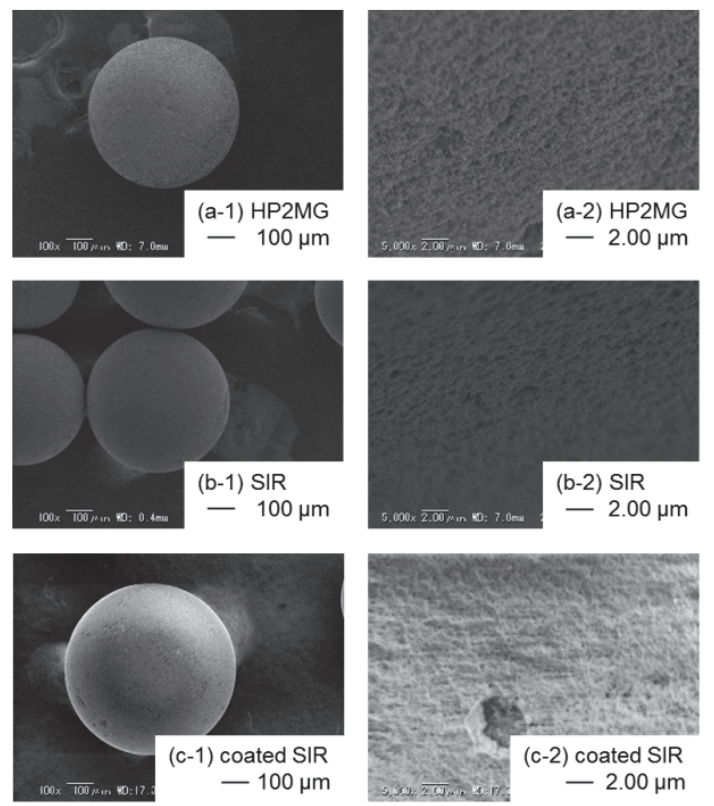

Fig. 2 Scanning electron micrographs of (a) HP2MG, (b) SIR, and (c) coated SIR with 3 wt $\%$ chitosan. (a-1) and (b-1) are from ref \#15.

\subsection{Batchwise adsorption of $\mathrm{Eu}^{3+}$ with SIRs}

The SIRs were applied to the adsorption of $\mathrm{Eu}^{3+}$. Figure 3 shows the effect of equilibrium $\mathrm{pH}$ on the adsorption amount of $\mathrm{Eu}^{3+}$. Adsorption of $\mathrm{Eu}^{3+}$ increased with increasing $\mathrm{pH}$ in all SIRs, indicating that the adsorption of $\mathrm{Eu}^{3+}$ progresses via the cation exchange reaction, similar to a conventional solvent extraction system using PC-88A.

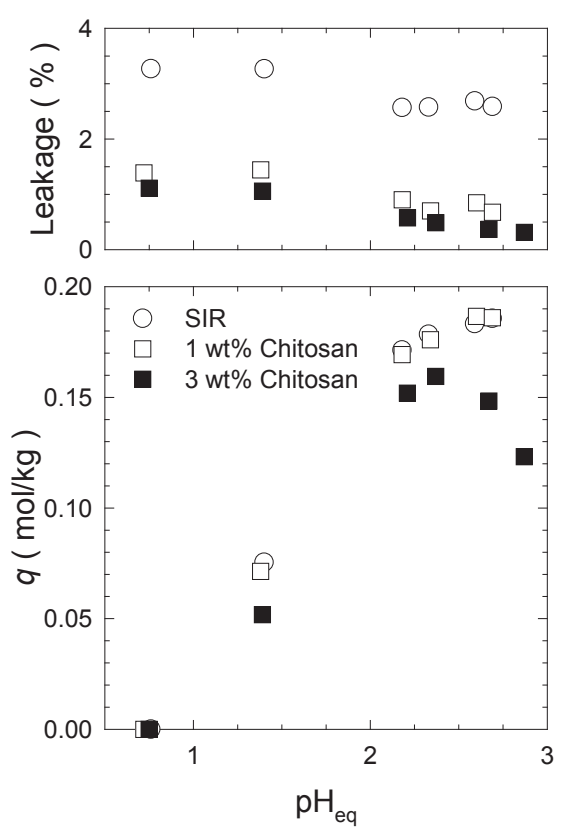

Fig. 3 Effect of equilibrium $\mathrm{pH}$ values on the adsorption amount of $\mathrm{Eu}^{3+}$ by $\mathrm{SIR}$ and coated SIRs in single $\mathrm{Eu}^{3+}$ system. $\left[\mathrm{Eu}^{3+}\right]_{\text {feed }}=1 \mathrm{mmol} / \mathrm{dm}^{3}$. 

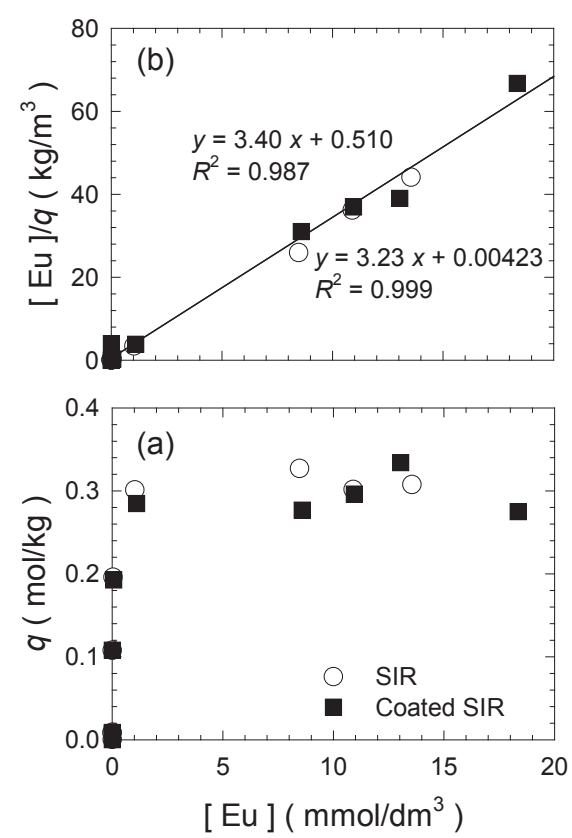

Fig. 4 (a) Adsorption isotherms for $\mathrm{Eu}^{3+}$ on uncoated and coated SIRs at equilibrium $\mathrm{pH}=2.27 \pm 0.05$ and (b) linearized Langmuir plots of the adsorption isotherms.

The adsorption of $\mathrm{Eu}^{3+}$ by coated SIR with $1 \mathrm{wt} \%$ chitosan was similar to that by SIR. The adsorption amount by coated SIR with $3 \mathrm{wt} \%$ chitosan was however decreased compared to those with SIR. In addition, the adsorption amount of $\mathrm{Eu}^{3+}$ with coated SIR with $3 \mathrm{wt} \%$ was decreased when the equilibrium $\mathrm{pH}$ was higher than 2.4. The decrease in the adsorption amount at higher $\mathrm{pH}$ region was not observed in the case of the coated SIR with crosslinked PVA ${ }^{15}$. The decrease in the adsorption amount may be due to interruption with the crosslinked chitosan. Leakage of PC-88A during batchwise adsorption is also shown in Fig. 3. In the case of SIR, ca. 3\% of PC-88A leaked during batchwise adsorption. The leakage of PC-88A was successfully reduced to less than $1 \%$ by coating of SIR with $3 \mathrm{wt} \%$ chitosan. The coating with $3 \mathrm{wt} \%$ chitosan was therefore an effective method for suppressing the leakage of the extractant, although the adsorption amount was slightly decreased. The SIR coated by $3 \mathrm{wt} \%$ chitosan would be shown as coated SIR hereafter.

Adsorption isotherms for $\mathrm{Eu}^{3+}$ with uncoated and coated SIRs were investigated at $\mathrm{pH}_{\mathrm{eq}}=2.27 \pm 0.05$. Figure $4 \mathbf{a}$ shows the adsorption isotherms. The adsorption isotherms of both adsorbents fitted well with the linear relationship of the Langmuir monolayer adsorption mechanism, as shown in Fig. 4b. The maximum adsorption amounts of $\mathrm{Eu}^{3+}$, obtained from the Langmuir adsorption isotherms were $0.310 \mathrm{~mol} / \mathrm{kg}$ for the uncoated SIR and $0.294 \mathrm{~mol} / \mathrm{kg}$ for the coated SIR, respectively. Maximum adsorption amount of $\mathrm{Eu}^{3+}$ on the coated SIR is found to be $95 \%$ of that on the uncoated SIR. Decrease in the maximum adsorption amount by coating can be suppressed compared to those with PVA crosslinked with boric acid ${ }^{13)}$ and with PVA crosslinked with glutaraldehyde ${ }^{15}$. Water-soluble chitosan therefore possesses higher potential as the polymeric film for coating SIR.

\subsection{Batchwise adsorption of $\mathrm{Eu}^{3+} / \mathrm{Y}^{3+}$ with coated SIR}

Batchwise adsorption of $\mathrm{Eu}^{3+} / \mathrm{Y}^{3+}$ with the coated SIR was then carried out in binary metal system, since these metals are contained in red phosphor materials. Figure $\mathbf{5}$ shows the effect of equilibrium $\mathrm{pH}$ on the adsorption amounts of $\mathrm{Eu}^{3+}$ and $\mathrm{Y}^{3+}$. Selective adsorption of $\mathrm{Y}^{3+}$ was observed, especially in low $\mathrm{pH}$ region. Adsorption of $\mathrm{Eu}^{3+}$ is however progressed when the equilibrium $\mathrm{pH}$ value is increased. The chromatographic operation should be used for the mutual separation of the metals. Decrease in the adsorption amount of $\mathrm{Y}^{3+}$ was also observed when the equilibrium $\mathrm{pH}$ was higher than 1.8. This is not however due to the interruption with the crosslinked chitosan, since total adsorption amount of $\mathrm{Y}^{3+}$ and $\mathrm{Eu}^{3+}$ was not decreased. The decrease in the adsorption amount of $\mathrm{Y}^{3+}$ in the binary system was therefore due to the limitation of adsorption capacity during the progress in the adsorption of $\mathrm{Eu}^{3+}$.

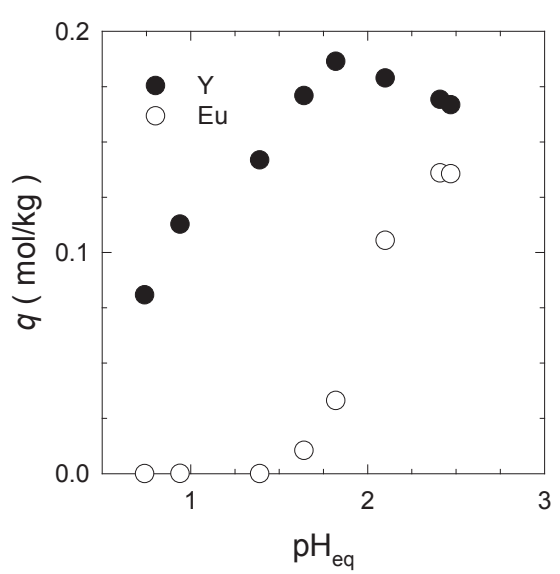

Fig. 5 Effect of equilibrium pH on the adsorption amount of $\mathrm{Eu}^{3+}$ and $\mathrm{Y}^{3+}$ in a binary metal system. $\left[\mathrm{Eu}^{3+}\right]_{\text {feed }}=\left[\mathrm{Y}^{3+}\right]_{\text {feed }}=1$ $\mathrm{mmol} / \mathrm{dm}^{3}$.

\subsection{Chromatographic separation of $\mathrm{Eu}^{3+} / \mathrm{Y}^{3+}$ with coated SIR}

The chromatographic separation of the metals using the coated SIR was firstly conducted using the conventional frontal analysis mode, as shown in Fig. 1a. Figure 6 shows the breakthrough and elution curves for $\mathrm{Eu}^{3+}$ and $\mathrm{Y}^{3+}$, from an aqueous feed solution containing $0.5 \mathrm{mmol} / \mathrm{dm}^{3}$ of $\mathrm{Eu}^{3+}$ and $13.3 \mathrm{mmol} / \mathrm{dm}^{3}$ of $\mathrm{Y}^{3+}$. Quantitative adsorption and elution could be carried out by the coated SIR. 

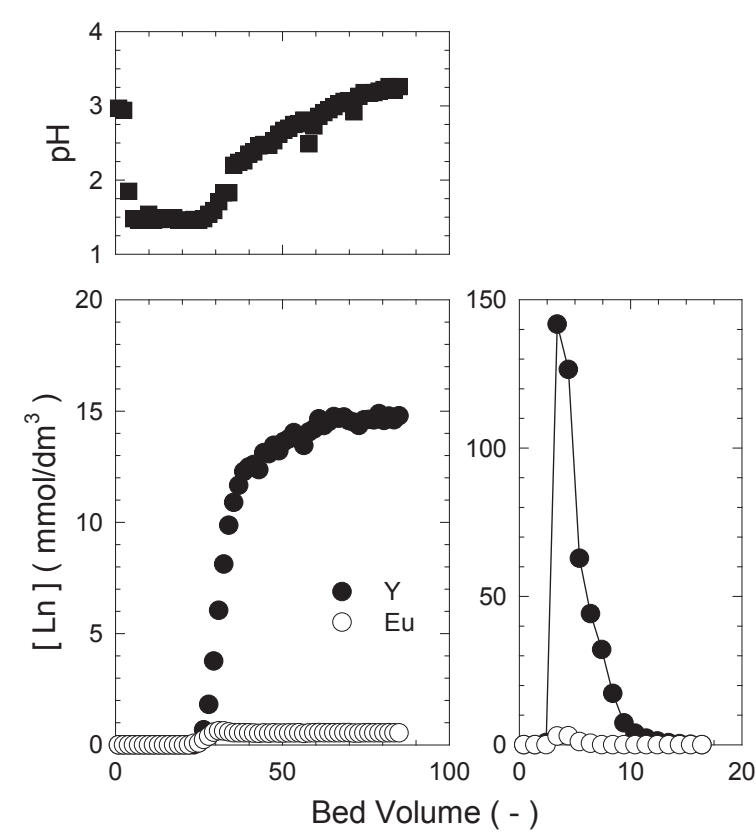

Fig. 6 (a) Breakthrough and (b) elution curves for $\mathrm{Eu}^{3+}$ and $\mathrm{Y}^{3+}$ from a binary metal solution by frontal analysis mode using the coated SIR. $\left[\mathrm{Eu}^{3+}\right]_{\text {feed }}=0.5 \mathrm{mmol} / \mathrm{dm}^{3},\left[\mathrm{Y}^{3+}\right]_{\text {feed }}=$ $13.3 \mathrm{mmol} / \mathrm{dm}^{3}$, and $\mathrm{pH}_{\text {feed }}=5.3$.

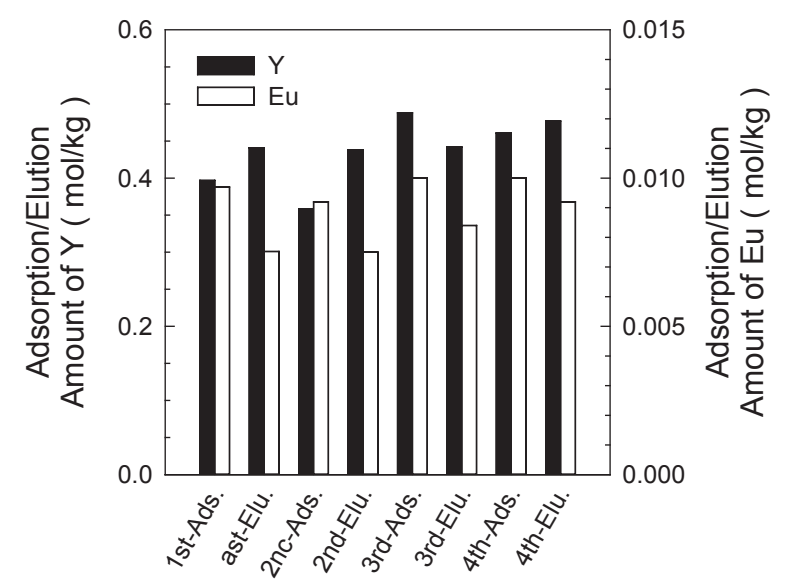

Fig. 7 Effect of repeated processing on the amounts of $\mathrm{Eu}^{3+}$ and $\mathrm{Y}^{3+}$ adsorbed and eluted using frontal analysis mode. $\left[\mathrm{Eu}^{3+}\right]_{\text {feed }}=0.5 \mathrm{mmol} / \mathrm{dm}^{3},\left[\mathrm{Y}^{3+}\right]_{\text {feed }}=13.3 \mathrm{mmol} / \mathrm{dm}^{3}$, and $\mathrm{pH}_{\text {feed }}=5.3$.

The reusability of the coated SIR, following the adsorptionelution processing, was also investigated using chromatographic frontal analysis under the same conditions as used in Fig. 6. The adsorption-elution was repeated 4 times. Similar breakthrough and elution curves to those in Fig. 6 for both metals, were obtained at all times. Figure 7 shows the amount of the metals adsorbed and eluted by repeated operation. These amounts of the metals adsorbed and eluted are scattered somewhat, owing to the graphical integration of the breakthrough and elution curves. However, since these amounts can be seen to maintain for 4 times adsorption-elution processing, the coated SIR possessed the sufficient loading and elution capacities for repeated operation.

Since the elution curves of the metals shown in Fig. $6 \mathrm{~b}$ were however overlapped, the mutual separation of the metals using the conventional frontal analysis mode was impossible. Gradient elution mode has been revealed to be effective to improve separation of the metals with chromatographic operation ${ }^{15,16)}$. The gradient elution was therefore conducted by connecting another column packed with the fresh coated SIR to the column which adsorption of the metals was saturated, and the loaded metals were eluted by dilute nitric acid with gradually increasing concentration. Figure 8 shows the elution curves of the metals. By using gradient elution mode with the development column, $\mathrm{Eu}^{3+}$ adsorbed was eluted in the beginning by dilute nitric acid, and the elution of $\mathrm{Y}^{3+}$ was started when the concentration of the nitric acid was increased. Complete separation between $\mathrm{Eu}^{3+}$ and $\mathrm{Y}^{3+}$ was therefore achieved by gradient elution mode. The peak for the elution of $\mathrm{Y}^{3+}$ was however splitted. This may be due to the possible turbulence of the eluent stream between the two columns connected.
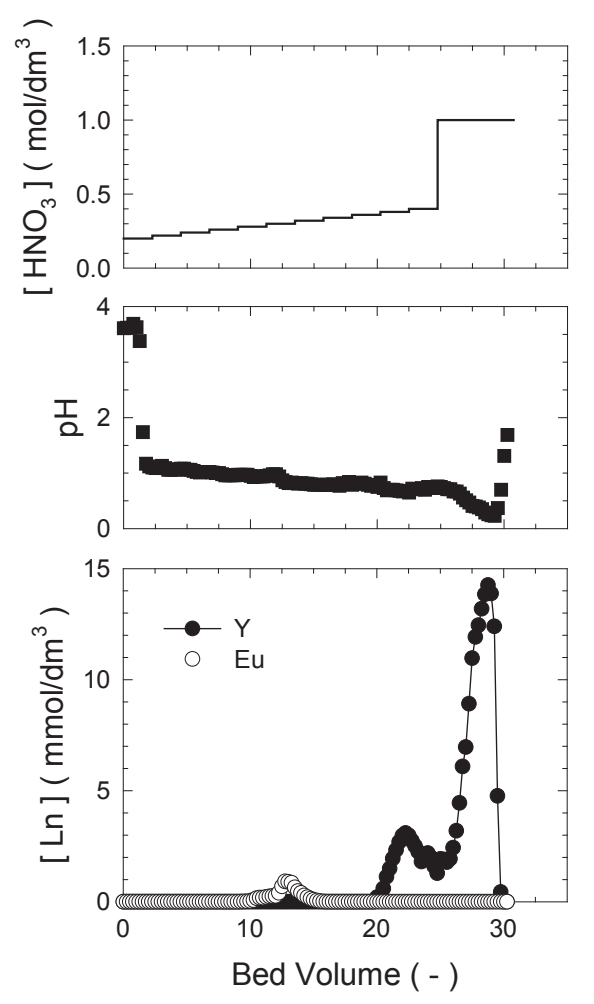

Fig. 8 Elution curves for $\mathrm{Eu}^{3+}$ and $\mathrm{Y}^{3+}$ by gradient elution mode. 


\section{Conclusion}

The SIR coated with crosslinked water-soluble chitosan was prepared and the separation of $\mathrm{Eu}^{3+}$ and $\mathrm{Y}^{3+}$ was investigated. The important features of the system are summarized as follows:

(1) Coating of the SIR with crosslinked water-soluble chitosan can suppress the leakage of PC-88A in SIR during adsorption. The adsorption of $\mathrm{Eu}^{3+}$ with the uncoated and coated SIRs follows the Langmuir adsorption mechanism and the maximum adsorption amounts at equilibrium $\mathrm{pH}=2.27$ were 0.310 $\mathrm{mol} / \mathrm{kg}$ for SIR and $0.294 \mathrm{~mol} / \mathrm{kg}$ for coated SIR. Decrease in the maximum adsorption amount by coating the SIR is much lower than other coated SIRs reported.

(2) The coated SIR can be applied in a chromatographic operation, and possesses sufficient loading and elution capacities for the repeated operation.

(3) Gradient elution mode with the development column, complete separation between $\mathrm{Eu}^{3+}$ and $\mathrm{Y}^{3+}$ can be achieved.

\section{Acknowledgement}

The authors are grateful to Prof. Kazuo Sakurai and Dr. Yusuke Sanada of The University of Kitakyushu for gel permeation chromatography measurement.

\section{References}

1) T. Nakamura, S. Nishihama and K. Yoshizuka, Solvent Extr. Res. Dev., Jpn., 14, 105 (2007).

2) F. Yang, F. Kubota, Y. Baba, N. Kamiya and M. Goto, J. Hazardous Mater., 254-255, 79 (2013).

3) T. Nakamura, T. Ikawa, S. Nishihama and K. Yoshizuka, Ion Exch. Lett., 2, 22 (2009).
4) M.P. Gonzalez, I. Saucedo, R. Navarro, M. Avila and E. Guibal, Ind. Eng. Chem. Res., 40, 6004 (2001).

5) R.S. Juang and M.L. Chen, Ind. Eng. Chem. Res., 36, 813 (1997).

6) C.Y. Shiau, C.L. Lin and H.S. Chang, Ind. Eng. Chem. Res., 44, 4771 (2005).

7) L.H. Reyes, I.M. Medina, R.N. Mendoza, J.R. Vazquez, M.A. Rodriguez and E. Guibal, Ind. Eng. Chem. Res., 40, 1422 (2001).

8) K. Onishi, T. Nakamura, S. Nishihama and K. Yoshizuka, Ind. Eng. Chem. Res., 49, 6554 (2010).

9) A.W. Trochimczu k, N. Kabay, M. Arda and M. Streat, React. Funct. Polymn., 59, 1, (2004).

10) S.P. Alexandratos and K.P. Ripperger, Ind. Eng. Chem. Res., 37, 4756 (1998).

11) S.P. Alexandratos and S.D. Smith, Solvent Extr. Ion Exch., 22, 713 (2004).

12) D. Muraviev, L. Ghantous and M. Valiente, React. Funct. Polymn., 38, 259 (1998).

13) Y. Yuan, J. Liu, B. Zhou, S. Yao, H. Li and W. Xu, Hydrometallurgy, 101, 148 (2010).

14) N. Kabay, J.L. Cortina, A. Trochimczuk and M. Streat, React. Funct. Polymn., 70, 484 (2010).

15) S. Nishihama, K. Kohata and K. Yoshizuka, Sep. Purif. Technol., 118, 511 (2013).

16) J. Shibata, S. Matsumoto, N. Nakayama, M. Mashimo, H. Yamamoto, Shigen-to-Sozai, 112, 873 (1996) (in Japanese). 